\title{
Achieving Valuable Weather and Climate Services
}

\author{
Alberto Troccoli
}

\begin{abstract}
Weather and climate services rely on the production and delivery of relevant, credible and, of course, valuable information. In this sense, the energy industry, with its long-standing and varied needs for these services and strong experience, provides a solid test bed for assessing these services. However, it is argued here that, whether for public or commercial use, weather and climate services are, in essence, no different to other more familiar services (e.g. financial). For weather and especially the more recent climate services to succeed, it is therefore important that lessons from these other common services - which also often deal with uncertain and complex information-are considered. It is also natural and important that the burgeoning climate services learn from the more mature and analogous weather services in order to leapfrog development. Initial public investment is critical to spur development of these services. Such investment should then be phased out in a managed way to avoid abruptly interrupting their growth phase and therefore jeopardising their sustainability, given the strong effort that is also being invested into developing these services. The criticalities of the weather and climate services - such as the accuracy and skill of the information-need to be borne in mind when modulating public investment.
\end{abstract}

A. Troccoli $(\bowtie)$

World Energy \& Meteorology Council, c/o University of East Anglia, Norwich, UK

(C) The Author(s) 2018

A. Troccoli (ed.), Weather \& Climate Services for the Energy Industry, https://doi.org/10.1007/978-3-319-68418-5_2 
Keywords Marketing approach - Innovation - Commercial value Financial service $\bullet$ Stakeholder $\bullet$ user $\bullet$ customer $\bullet$ Transparency Trustworthiness

\section{What's a SERVICE- \\ Never Mind the Weather and Climate?}

Whether for public good or commercial use, the value of weather and climate information is ultimately measured in terms of its usefulness to society and, specifically, for the energy sector in our case. The way in which this information is conveyed can be multifaceted-it may be in the form of a temperature map for instance-but in order for it to be most effective it needs to be 'packaged' in terms of a service. Although the definition of service can vary depending on the objective, scope and maturity of the information or product, the overall aim of a service is to meet the requirements of the user of the service, by extracting the highest value from, in our case, weather and climate information for the specific application at hand, be it the forecasting of hydropower production or the impact of a snowstorm on the infrastructure used for electricity transmission.

Thus, in its most general sense, a service can be defined as:

A set of actions aimed at helping its beneficiaries make the best use of tailored information so as to improve their 'business'.

It is therefore apparent that a 'weather or climate' service is not unique, amongst other possible services. Although one can attempt a specific definition of weather or climate service, ${ }^{1}$ it is useful to first try to understand how a weather/climate service differs from, say, a financial service, or from a medical service, or even a car service. These may look like disparate analogies but assessing these can help understand better what a weather/ climate service is and what it is supposed to achieve. More specifically, we are arguing here that although each of these services delivers different outputs and outcomes, there is no fundamental distinction between them, in the sense of the above definition.

These services naturally differ from each other in terms of their specific features. If, for example, we characterise a service based on the following four features, ${ }^{2}$ 
- Maturity: how long have they been around?

- Tangibility: is it something we can easily relate to?

- Level of Risk: how reliable and/or accurate is the output/product?

- Trustworthiness (or Credibility): how much do we trust the service provider?

it is possible to categorise and inter-compare them as presented in Table 2.1. What this comparison tells us is that: (1) there is indeed nothing special about a weather/climate service from a conceptual point of view as they can easily be related/compared to other, more traditional and widespread, services; (2) weather and, especially, climate services carry a higher level of risk or 'caution' (e.g. of the likelihood or certainty of climate forecast) than the other comparator services. It should indeed be the focus of the experts involved in the development of weather and climate services to attempt to reduce these levels of 'cautioning' as much as possible. The next section provides some indication on how this 'caution' reduction may be achieved.

\section{Public versus Commercial Approach-How Does a Service Differ in These Two Contexts?}

Before looking more closely at how the 'caution' level can be reduced, it is important to reflect a little more on the analogy amongst these services. One seemingly important feature, which was deliberately omitted in the comparison presented in Table 2.1, is the nature of the service. In other words, is the service commercial and/or a public good (including scientific) service? Although this feature appears to be critical, the reason it has

Table 2.1 Qualitative comparison between five different types of service based on the four representative features-Maturity, Tangibility, Level of Risk, Trustworthiness

\begin{tabular}{|l|c|c|c|c|}
\hline & Maturity & Tangibility & $\begin{array}{c}\text { Level of } \\
\text { Risk }\end{array}$ & Trustworthiness \\
\hline Financial Service & $\mathrm{H}$ & $\mathrm{M}$ & $\mathrm{M}$ & $\mathrm{M}$ \\
\hline Medical Service & $\mathrm{H}$ & $\mathrm{H}$ & $\mathrm{M}$ & $\mathrm{H}$ \\
\hline Car Service & $\mathrm{H}$ & $\mathrm{H}$ & $\mathrm{L}$ & $\mathrm{M}$ \\
\hline Weather Service & $\mathrm{H}$ & $\mathrm{M}$ & $\mathrm{M}$ & $\mathrm{M}$ \\
\hline Climate Service & $\mathrm{L}$ & $\mathrm{L}$ & $\mathrm{H}$ & $\mathrm{M}$ \\
\hline
\end{tabular}

The three qualitative levels- $-\mathrm{H}, \mathrm{M}$ and L-stand for High, Medium and Low, respectively. These are associated with colours to indicate the level of 'caution'. So, for instance, a high maturity (e.g. > 30 years) carries a low level of cautioning (green) whereas a high risk carries a high level of cautioning 
been omitted in Table 2.1 is simple: the distinction between commercial (or private) good and public good, it is argued here, is essentially irrelevant if the aim is to deliver an effective service. Indeed, if a service is to be successfully adopted, it needs to be developed, promoted and implemented in a very similar way, irrespective of whether the service is commercial or public in nature. Indeed, while the way in which these three activities-development, promotion and implementation-is carried out may differ in the two contexts, they remain critical in both the commercial and public domains.

Another way to look at this is that lessons learnt in one domain can be transferred to the other. Thus, for instance, it is likely that a more commercially focussed 'traditional' sales approach can lead to an improved and more highly adopted public (weather/climate) service. Specifically, a sales approach would be based on the following four personal (i.e. human) characteristics or traits:

- Eloquence-To be able to influence the other person's decision about their need for the service

- Cultural awareness-To be able to relate to different people regardless of their backgrounds, gender, religion and so on.

- Flexibility-To be able to operate outside of day-to-day routine in order to better tackle new or unexpected situations

- Transparency and honesty-To critically believe in what one is promoting

Of the four traits, the last one is by far the most critical. If you cannot convince yourself that something is valuable and useful, having properly appraised its pros and cons, it is going to be very hard to convince others. In other words, you need to be genuinely convinced that the service is worth buying, and that you would actually buy it yourself-ideally, you have already bought it! And the best way to convince yourself is to be as transparent and honest as possible. ${ }^{3}$

While sales approaches differ, one should make the most use of the huge amount of knowledge accrued in the marketing arena (Aaker and McLoughlin 2010; Homburg et al. 2012), irrespective of the service or product for which these approaches were devised. And thinking that a public good service should be treated differently just because the user will not be directly charged - remember they would have already paid for it via taxation-can be a serious mistake, particularly if the service development process is led by non-commercially savvy people. 
As remarked above, key to the uptake of a service is the careful development, promotion and implementation of a service. This process requires close interaction with the final users of the service. The aim of this interaction is to attentively capture the specifications of the service while also advising on the potentials a service can offer (e.g. see also Chaps. 3 and 4 , for further discussion, especially in the context of the energy industry). Moreover, this process should be carried out in an iterative way-namely through regular consultations with the users. Overall, rigour and meticulousness need to be applied in the development of the service. Ultimately this approach will ensure the production of a useful, robust and longlasting, and ideally replicable, service/product.

\section{Adding Weather and Climate to the Service}

Having drawn some analogies between meteorological (namely weather and climate) services and other more commonly known services, we here discuss how these analogies can be harnessed to produce better services for weather and climate. To a large extent, the discussion here prescinds from the source of funding required to develop meteorological services and from the quantification of the economic benefits of such services. Although these are very important discussions, they have extensively been authoritatively discussed in the literature (Freebairn and Zillman 2002a; Freebairn and Zillman 2002b; WMO 2015).

In spite of the close similarities between weather and climate services, there are three important distinctions to be drawn between weather services and climate services, leaving aside their intrinsic distinction according to which weather services essentially deals with forecasts of up to a few weeks in advance, and climate services deals with forecasts and projections from a few weeks to decades. ${ }^{4}$ In terms of services, their main differences are:

1. Weather services are considerably more mature than climate services; the former have been around for 40+ years (Pettifer 2015), the latter have started to be developed in a consistent way only during the last decade (Hewitt et al. 2012);

2. Weather services are based on information (e.g. forecasts) that are both more accurate (shorter lead time) and verifiable (their lifespan is shorter, in line with its lead time) compared to climate information $^{5}$

3. Data (including forecasts) policy for weather services can be different, and more restrictive, than that for climate services ${ }^{6}$ 
How these differences translate in practice is that weather services have accrued a strong basis, both in terms of products and (their related) market. From the low levels of the 1970s, European weather services now have an estimated value of $€ 300$ million per annum (excluding aviation) (Pettifer 2015). In addition, Lazo et al. (2011) estimated that US economic activity that is attributable to weather variability could be $3.4 \%$, or $\$ 485$ billion of the 2008 gross domestic product. It is therefore apparent that the weather services market-whether supplied by National Meteorological Services (NMS) or private sector providers-has a solid foundation.

This also means that users (or customers) of weather services are normally well aware of the potential and usefulness of weather products and therefore a smaller effort is required to persuade them compared to when climate products are promoted. For instance, there are established meetings for users, such as the European Centre for Medium-Range Weather Forecasts (ECMWF)'s 'Using ECMWF's forecasts' (UEF), ${ }^{7}$ as well as a host of private weather service companies that are working to improve the customisation of weather products for their customers. While the cost of the basic weather information (typically the forecasts) charged by some NMS (as is the case for most European NMSs) has been an important barrier for start-up enterprises (Pettifer 2015), the market for weather services has been such that this barrier has been overcome by a large number of companies. ${ }^{8}$

Different is the case for climate services, for which the accuracy (or skill) of the product still plays a crucial limiting factor in their uptake. ${ }^{9} \mathrm{~A}$ distinction needs to be made, however, between the climate forecasts, which have lead times from a few months to a year (also referred to as seasonal forecasts), and climate outlooks and projections, which consider time horizons from a few years to multi-decades ahead (referred to as decadal outlooks, over the following decade, and climate projections, beyond it).

In the case of seasonal forecasts, some regions of the world have some useful skill, namely they can be predicted more accurately than others (typically tropical areas), while others have little or no skill at all (normally regions at higher latitudes, like Europe, e.g. see Troccoli et al. 2008; Troccoli 2010), the skill being also dependent on the season and, particularly, the variable (air temperature usually has a higher skill than precipitation, or wind speed or solar radiation). It can therefore be difficult to convince a prospective user or customer to make use of such forecasts in 
areas or for variables that traditionally do not display an improvement in skill compared to using a long-term mean or climatology even if there is increasing evidence that their level of skill is improving (Alessandri et al. 2017). Thus, interest in and uptake of seasonal forecasts are increasing. Work on the applications of seasonal forecasts, initially spearheaded by the International Research Institute for Climate and Society (IRI), ${ }^{10}$ is now becoming mainstream thanks to activities carried out at organisations such as the APEC Climate Center (APCC) ${ }^{11}$ or programmes such as the Copernicus Climate Change Services (C3S). ${ }^{12}$

In the case of climate projections, it is essentially impossible to prove their level of accuracy. The best that can be done is to demonstrate the suitability of the climate models at representing features at the country or sub-country level (and not just global or regional averages). This can mainly be done on the basis of the climate model performance over the recent (observed) climate period.

From an application point of view, seasonal climate forecasts are relevant for operational matters such as resource management and infrastructure maintenance scheduling while climate projections are relevant for infrastructure planning purposes. Indeed, it is because of the increasing interest in these applications, with energy providing a prime example, that seasonal forecasts and climate projections are continually being developed and tailored to an increasing number of (prospective) users or customers (see also Chap. 12).

In spite of these advances, there remains the strong need for a close interaction with prospective users in order (1) to improve the service producer's understanding of the final use of the climate information so that it can be appropriately tailored and (2) for the user to better appreciate the strengths and limitations of the climate information. These are processes that require substantial time investment, both on the relationship building side and on the technical development side. Most important of all is the ultimate instillation of confidence in prospective customers in relation to climate services. This instillation does not mean over-selling the services, rather that one must extract the most information relevant for the specific application knowing the limitations of the product-remember the key marketing trait is 'Transparency and honesty' section 'Public versus Commercial Approach-How Does a Service Differ in These Two Contexts?'. Weather services have gone through this process much before climate services and so lessons could be learned from this experience, bearing in mind the fundamental difference 
between the two services, namely the level of accuracy and skill of the products. Lessons can also be learned by analogy from other services, as argued above particularly by adopting relevant commercial marketing techniques.

This type of approach is also supported by Brooks (2013) who argues that we now have the opportunity to provide innovative climate services (new datasets and products) to climate-sensitive sector clients. The key to achieving relevant, valuable and discontinuous climate services is to accelerate innovation in climate services, and decisively cross the Research-to-Operation valley of death. This, Brooks (2013) argues, can be achieved by adopting three Es-Engagement, Entrepreneurship and Evaluation.

It is sometimes argued that climate services should be mainly a public good exercise (e.g. Webber and Donner 2016). This view appears shortsighted since, as argued in this chapter, development of climate services can greatly benefit from trying to adopt a commercial approach. As these services mature, and the commercial value of climate services becomes more apparent, opportunities develop, and there will be an ever larger share of services that are offered at a cost. Thus the route to achieving a sustainable climate service is to embrace a standard, market-oriented approach, also expressed by the three Es of Brooks (2013). Of course, one should test whether it is more efficient, or cost-effective, to run a public good service (i.e. with public funding) or a commercial one. It can be easy to forget that public good services require substantial investment. This investment needs of course to be weighed against their benefit. More generally, while an initial public investment is beneficial, and even required, in order to set in motion a (commercial) activity, it is important to scale back public funding or subsidies (e.g. as done in the case of feed-in-tariff for solar photovoltaic installations in a number of countries), but in a measured and reasoned way. One should also avoid falling into the trap of heavily funding a 'commercial' entity through public grants thinking that the services developed with these grants have value just because that commercial entity is involved in the development of the services. While such development may be useful to create capacity and eventually have a commercial entity which is self-reliant, it is entirely possible that reliance on public funding makes the 'commercial' entity risk-averse and therefore never really self-sustaining. 


\section{SUMMARY}

The development of weather and climate services should have as their ultimate objective the achievement of the best quality information to serve both the commercial and the public worlds. While weather services are well established, both in a commercial sense and in a public good way (e.g. through the weather forecasts regularly issued by media outlets), climate services can benefit from the lessons learnt by weather services, but also other analogous services (e.g. financial or medical services). Central to this analogy is the marketing approach and greater focus on the needs of specific customer groups, which at its heart requires an honest and transparent approach for it to be most effective in the long run. Having to deal with a product - climate information-which is highly uncertain and with relatively low skill, there is a strong need to create an environment of trust. Without this there is a risk to build a sand castle, which at the first high tide will be washed away.

Thus, the key to building valuable and self-sustaining (weather and climate) services is to grow confidence in the products by cultivating personal relationships with prospective users/customers. Achieving this objective requires substantial time and funding investment, particularly in the case of the newer climate services (compared to weather services). This investment should focus on providing strengthened education, training and knowledge transfer activities, as these are key components of enhancing confidence in a product/service.

Of course, investment to also improve and refine the underlying meteorological data and products is fundamental. Public funding that is being invested to develop these (climate) services, as in the case of C3S or the European Union H2020 programme, is therefore essential in this phase of development. Specifically, projects such as the European Climatic Energy Mixes (ECEM), ${ }^{13}$ which is building a climate and energy demonstrator for the energy sector, are trying to make the best use of this funding by building a service for both commercial and public users, through a strong engagement with stakeholders.

The key question then is 'how much, and for how long, public investment should be used in order to achieve the best possible weather and climate services from both a public and commercial point of view?' While there is no easy answer, a good guide to addressing this question is to keep in mind the critical factors in the development of these services 
(e.g. accuracy and skill of products). Better understanding and analysing this key question could be an area of further investigation.

Acknowledgements The author would like to acknowledge two reviewers for valuable input to this chapter.

\section{Appendix-Definitions of Climate Service}

It is only recently that the climate community has got to grips with the concept of 'service'. As a consequence, this is still a relatively new concept, and that is perhaps why its definition is varied-here we present a few, verbatim, just to illustrate the point.

\section{The Global Framework for Climate Services Definition}

Climate services provide climate information in a way that assists decision making by individuals and organisations. Such services require appropriate engagement along with an effective access mechanism and must respond to user needs. Such services involve high-quality data from national and international databases on temperature, rainfall, wind, soil moisture and ocean conditions, as well as maps, risk and vulnerability analyses, assessments and long-term projections and scenarios. Depending on the user's needs, these data and information products may be combined with nonmeteorological data, such as agricultural production, health trends, population distributions in high-risk areas, road and infrastructure maps for the delivery of goods, and other socio-economic variables.

http://www.gfcs-climate.org/what_are_climate_weather_services

\section{The Climate Service Partnership Definition}

Climate services involve the production, translation, transfer and use of climate knowledge and information in climate-informed decision making and climate-smart policy and planning. Climate services ensure that the best available climate science is effectively communicated with agriculture, water, health and other sectors, to develop and evaluate adaptation strategies. Easily accessible, timely and decision-relevant scientific information can help society to cope with current climate variability and limit the economic and social damage caused by climate-related disaster. Climate services also allow society to build resilience to future change and take advantage of opportunities provided by favourable conditions. Effective 
climate services require established technical capacities and active communication and exchange between information producers, translators and user communities.

http://www.climate-services.org/about-us/what-are-climate-services/

\section{The Climate Europe Definition}

A climate service is the provision of climate information to assist decision making. The service must respond to user needs, must be based on scientifically credible information and expertise and requires appropriate engagement between the users and providers. Policy makers can use climate services to access decision-relevant scientific information in order to make the best decisions for society as a whole. This can help society to cope with current climate variability and limit the economic and social damage caused by climate-related disasters. Climate services ensure that the best available climate science is effectively communicated with agriculture, water, health and other sectors, to develop and evaluate adaptation strategies.

https://www.climateurope.eu/definitions-climate-services/

\section{Notes}

1. For climate services, several definitions exist, sometimes even circular; a few of them are presented in the Appendix.

2. Other helpful features could be identified, such as usefulness of, or value of or share of the population affected by the service, but the discussion here is not intended to be exhaustive, merely illustrative.

3. We say 'as far as possible' because there is no need for instance to disclose and discuss technical details, such as an approximation applied in a programming code used to produce a given map, as long as we genuinely think this is not affecting the overall message of the map.

4. The exact boundary between weather and climate services is sometimes blurred.

5. While seasonal forecasts can be verified, although not as frequently as weather forecasts, climate projections cannot, at least not in a strong statistical sense due to very long time horizon compared to the human lifetime and their limited sample.

6. While there are common data policies on common data sharing, data policies for weather forecasts differ substantially in say USA (free sharing) and Europe (data are charged). For further discussion, see Harrison and Troccoli (2010) or WMO (2015). 
7. https://www.ecmwf.int/en/learning/workshops.

8. More needs to be done to completely remove or at least further reduce this barrier, however, as experience shows that an open-data policy tends to lead to a dramatic increase in the use of the data (Pettifer 2015, see also World Bank 2017).

9. Climate (and weather) services are here mainly treated in terms of predictions/projections. Historical (past) data could also be included but, while they might technically belong to climate services, they can be found in both weather and climate services.

10. http://iri.columbia.edu/.

11. http://www.apcc21.org.

12. http://climate.copernicus.eu/.

13. http://ecem.climate.copernicus.eu/.

\section{REFERENCES}

Aaker, D., \& McLoughlin, D. (2010). Strategic market management: Global perspectives (1st ed.). Chichester: John Wiley \& Sons, 368 pp.

Alessandri, A., De Felice, M., Catalano, F.,.Lee, J.-Y., Wang, B., Lee, D. Y. et al. (2017). Grand European and Asian-Pacific multi-model seasonal forecasts: Maximization of skill and of potential economical value to end-users. Climate Dynamics. https://doi.org/10.1007/s00382-017-3766-y.

Brooks, M. (2013). Accelerating innovation in climate services. Bulletin of the American Meteorological Society, 94, 807-819.

Freebairn, J. W., \& Zillman, J. W. (2002a). Economic benefits of meteorological services. Meteorological Applications, 9, 33-44.

Freebairn, J. W., \& Zillman, J. W. (2002b). Funding meteorological services. Meteorological Applications, 9, 45-54.

Harrison, M., \& Troccoli, A. (2010). Data headaches. In A. Troccoli (Ed.), Management of weather and climate risk in the energy industry. NATO Science Series (137-147). Dordrecht: Springer Academic Publishers.

Hewitt, C., Mason, S., \& Walland, D. (2012). The global framework for climate services. Nature Climate Change, 2, 831-832.

Homburg, C., Kuester, S., \& Krohmer, H. (2012). Marketing management - a contemporary perspective (2nd ed.). London: McGraw-Hill Education, 641 pp.

Lazo, J. K., Lawson, M., Larsen, P. H., \& Waldman, D. M. (2011). U.S. economic sensitivity to weather variability. Bulletin of the American Meteorological Society, 92, 709-720. https://doi.org/10.1175/2011BAMS2928.1.

Pettifer, R. (2015). The development of the commercial weather services market in Europe: 1970-2012. Meteorological Applications, 22, 419-424.

Troccoli, A. (2010). Seasonal climate forecasting: A review. Meteorological Applications, 17, 251-268. https://doi.org/10.1002/met.184. 
Troccoli, A., Harrison, M., Anderson, D. L. T., \& Mason, S. J., eds. (2008). Seasonal climate: Forecasting and managing risk. NATO Science Series. Dordrecht: Springer Academic Publishers., 467 pp.

Webber, S., \& Donner, S. D. (2016). Climate service warnings: Cautions about commercializing climate science for adaptation in the developing world. WIREs Climate Change. https://doi.org/10.1002/wcc.424.

WMO. (2015). Valuing weather and climate: Economic assessment of meteorological and hydrological services. World Meteorological Organization, 1153, $308 \mathrm{pp}$. Retrieved from http://www.wmo.int/pages/prog/amp/pwsp/documents/ wmo_1153_en.pdf

World Bank. (2017). Open Government Data Toolkit. http://opendatatoolkit. worldbank.org

Open Access This chapter is distributed under the terms of the Creative Commons Attribution 4.0 International License (http://creativecommons.org/licenses/ by $/ 4.0 /$ ), which permits use, duplication, adaptation, distribution and reproduction in any medium or format, as long as you give appropriate credit to the original author(s) and the source, a link is provided to the Creative Commons license and any changes made are indicated.

The images or other third party material in this chapter are included in the work's Creative Commons license, unless indicated otherwise in the credit line; if such material is not included in the work's Creative Commons license and the respective action is not permitted by statutory regulation, users will need to obtain permission from the license holder to duplicate, adapt or reproduce the material.

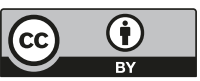

